\title{
Role of oxytocin in improving the welfare of farm animals - A review
}

\author{
Siyu Chen ${ }^{1 * *}$ and Shusuke Sato ${ }^{2}$
}

\section{* Corresponding Author: Siyu Chen Tel: +81-0229-84-7377, Fax: +81-0229-84-7377 E-mail: jihebuluo2015@163.com \\ 1 Graduate School of Agricultural Science, Tohoku University, Miyagi 981-8555, Japan \\ ${ }^{2}$ Animal Science, Faculty of Life and Environmental Science, Teikyo University of Science, Tokyo 120 0045, Japan}

Submitted Dec 25, 2015; Revised Feb 15, 2016; Accepted Feb 29, 2016

\begin{abstract}
Recently, increasing attention has been paid to the welfare of farm animals, which have been evaluated using behavioral and physiological measures. However, so far, the measures have almost always been used to estimate poor welfare. In this review, firstly we focus on how oxytocin (OT) relates to positive social behavior, pleasure, and stress tolerance, and second on which management factors stimulate OT release. OT induces maternal and affiliative behaviors and has an anti-stress effect. Further, OT is produced during enjoyable events, and has positive feedback on its own release as well. Therefore, to some extent, the relationship of OT to positive normal behavior was mutually beneficial-heightened OT concentration owing to comfortable rearing conditions induces positive social behavior, which in turn may increase OT concentration. Hence, studies on animal welfare should pay more attention to increasing comfort and the stress tolerance, rather than only focusing on when stress occurs in farm animals.
\end{abstract}

Keywords: Behavior; Farm Animals; Management; Oxytocin; Welfare

\section{INTRODUCTION}

Recently, the topic of animal welfare has been paid more and more attentions. In order to improve the welfare of farm animals, studies have been carried out using several methods, including behavioral and physiological measures. Of the behavioral measures, stereotypies [1] have been widely used as behavioral indicators of poor welfare in addition to escape behavior [2] and so on. On the other hand, activation of the hypothalamic-pituitary-adrenal (HPA) system and the sympathetic-adrenal-medullary system has been widely used as physiological indicators of poor welfare in human and animal experiments. Most welfare assessments have been conducted when the animals were under stressful conditions. Duncan and Olsson [3] advocated that freedom from the state of suffering is assured by providing environmental requirements, while the establishment of pleasurable states requires environmental enrichment. Thus, studies on animal welfare are gradually moving from merely the reduction of stress to methods of increasing pleasure and stress tolerance in the animals lifetime. Consequently, assessments of good welfare are becoming increasingly important. Under these circumstances, affiliative behavior and positive social interaction have been used as good welfare indicators. In addition, dopamine, endorphin, and serotonin have been used as indicators to evaluate good animal welfare. However, it has been pointed out that the release of dopamine induces the release of catecholamine [4], which is a stress indicator, whereas endorphin and serotonin influence sedation rather than pleasure and have no particular correlation with behavior $[5,6]$.

Interestingly, it was reported that hormone oxytocin (OT) mediate not only the affiliative and social behaviors related to good welfare mentioned above, but also responses to pleasurable events $[7,8]$. Thus, OT as a good welfare indicator [9] has recently gained 
importance in this topic [10].There are many reviews on the topic of animal welfare. The concept of animal welfare has been discussed based on viewpoints of theoretical foundation and its increasing developments $[11,12]$. Even if those were reviewed with animal production, those were almost based on the critical aspects [13-15].

We tried to review OT studies from the viewpoint of increasing welfare in farm animals. In this review, we focus on how OT, as a good welfare indicator, is playing roles on the welfare of farm animals, and what kind of animal management increases OT concentration.

\section{ROLES OF OXYTOCIN ON ANIMAL WELFARE}

\section{Role of oxytocin on positive social behavior}

OT, discovered in 1909, had been studied as a hormone involved in milk ejection and uterine contractions. It is produced by two kinds of oxytocinergic neurons: The magnocellular neurons sending their projections to the neurohypophysis releasing OT into blood circulation, and the parvocellular neurons sending their projections mainly to the campus, ventral tegmental area, frontal cortex, brainstem, pons, medulla, and spinal cord [16].

According to Ross and Young [8], in the late 1970s, pharmacological studies began to reveal a role for OT not only in birth and lactation, but also in synchronizing maternal behavior. During pregnancy and parturition, mothers nurture the fetus and protect the offspring after birth; this induces a series of hormonal changes in the mothers and offspring. Subsequently, Pedersen and Prange [17] firstly indicated the effect of OT on maternal behavior in rats. The study showed that 6 of 13 animals in the group administered intracerebroventricular OT became fully maternal (including 5 items designed by authors), whereas none of the 12 animals in the saline control group became fully maternal. Thus, the role of OT in maternal behavior began to receive more attention. Daily brief separation from pups induced maternal behaviors in dams, which heightened the sensitivity of OT receptors in the brain [18]. Further, the effect of the administration of OT on the incidence of maternal behavior in virgin rats was summarized by Pedersen et al [19], though not all studies demonstrated OT-induced maternal behavior. OT secretion from the paraventricular nucleus also induced maternal behavior in sheep [20]. However, this effect has not been ascertained in cattle [21].

Maternal behavior includes licking, grooming, and nursing of the offspring, and plays an important role in supporting the life of the offspring. From the above, it is evident that studies on OT in relation to maternal behavior in farmed animals are important for improving the welfare and survivability of the offspring.

The relationship of OT with maternal behavior with reference to the pair-bond between mothers and offspring, caused interest among researchers about the mechanism by which OT might mediate behavior in pair bonding. One of the first reports in this regard indicated that centrally administered OT induced affiliative behavior and facilitated bonds in female prairie voles [22]. Additionally, [23] indicated that affiliative behaviors might induce hypothalamic OT expression, which in turn may attenuate stress responses in delayed gastric emptying and accelerated colonic transit. These results are supported by those of Dhakar et al [24], who showed that OT-receptor knockout rats performed exhibited aggressive behavior than intact animals. Further, centrally administered OT also induced grooming behavior in male rats [25], affiliative response of huddled sitting in the subordinate squirrel monkey [26], and flexible behaviors such as locomotors activity in the open field under stress condition [27].

Dunbar [28] reported that social grooming, as an affiliative behavior, plays a particularly important role in social bonding and has a major effect on an individual's lifetime reproductive fitness in humans and primates. Social grooming in cows plays a role in functional significance for the formation and maintenance of social bonds and the stabilization of social relationships [29].

As a consequence, the evaluation of OT with relation to positive social behavior, such as maternal and affiliative behavior, may contribute greatly towards improving the welfare and fitness of farm animals.

\section{Role of oxytocin on positive emotion}

OT has been examined in relation to pleasurable events and positive interactions. For example, massage-like stroking of the abdomen of anaesthetized rats lowered blood pressure, and this effect was diminished by intravenous injection of an OT antagonist [30]. Morhenn et al [31] reported that comfortable physical contact increased endogenous OT concentration in human. Their results indicated that serum OT increased during massage followed by trust, but did not increase either with trust or with massage alone, suggesting that increased OT concentration is associated with pleasurable emotion. Further, the supportive interaction "warm touch" between married couples increased their salivary OT concentration [32]. In calves, serum OT concentration was higher in the natural suckling rearing system than in bucket-suckling calves [33]. In sows, Algers et al [34] reported that stimulation of the udder by piglets increased OT levels.

The OT-mediated pleasurable emotion described above may be a response to sensory activation. Uvnäs-Moberg [7] reported that social behavior and affiliative interaction involves sensory stimuli capable of releasing OT, which stimulates the attachment of bonding and in turn induces more OT release.

\section{Role of oxytocin on stress tolerance}


OT was involved in the HPA axis in exerting an anti-stress effect. It was reported that intranasal OT administration attenuated the adreno-cortico-tropic-hormone. response in monkeys [35], and centrally administered OT attenuated cortisol secretion in Holstein steers [36]. Further, intranasal OT injection had an anxiolytic-like effect on rats $[37,38]$. Thus, since the modern intensive livestock industry comprises many stressors to animals, studies on the anti-stress effects of OT should be paid more attention.

\section{Role of oxytocin on positive feedback effects}

Moos et al [39] found that intraventricular injection of OT induced a dose-dependent rise in OT release from the nuclei of both male and lactating rats, whereas an OT antagonist significantly reduced basal OT release in magnocelluar nuclei. These findings suggest that OT has a positive feedback effect on its own release. Da et al [20] (1996), noting that central OT-induced maternal behavior in sheep, also suggested that OT exerted positive feedback, as the intracerebroventricular administration of OT induced maternal behavior, which in turn facilitated OT release in both of central terminal and peripheral into the blood. In addition, Neumann et al [40] reported that OT exerts positive feedback in the supraoptic nucleus during suckling, suggesting that natural suckling may increase basal serum OT concentrations in rats. Although the positive feedback of OT has not been reported during other behaviors so far, it may be exerted not only during suckling and maternal behavior but also during other positive normal behaviors associated with pleasant feelings. Owing to this, we postulate that OT and positive social behavior and positive emotion is mutually beneficialOT concentration induces positive social behavior and positive emotion, which in turn increases OT concentration. The heightened OT concentration may be beneficial to animals, since it increases the stress tolerance [7] (Uvnäs-Moberg 1998) and engenders positive emotional states [41].

\section{MANAGEMENT PRACTICES FOR INCREASING OT CONCENTRATION IN ANIMALS IN ANIMAL HUSBANDRY}

An instantaneous increase in serum OT concentrations has been reported in calves during natural suckling that lasts only for $1 \mathrm{~min}$ [42]. In our previous study, we found that the basal serum OT concentration in 1-month-old calves was higher under the natural suckling system than the bucket-suckling system [33]. We speculated two possible causes for this finding. Heightened serum OT concentration might be due to the positive feedback by instantaneous secretion, firstly during long-term natural suckling, similar to results obtained with rats [40] and secondly during physical interactions with dams and peers. This postulated might be in agreement with that of Odendaal and Meintjes [43] that positive behavioral interactions stimu- late OT secretion in both humans and dogs. Hence, from these views, the welfare of farm animals would be improved under the natural suckling rearing system via an increase in serum OT concentration. This may be supported by the arguments of Krohn [44], who concluded that natural suckling systems, where calves are nursed by dams and have social contact with other calves and cows, are more beneficial to calf welfare, with results such as a greater positive effect on daily gain and vitality of the calf than is seen in the artificial rearing systems. These results might be partly mediated by OT.

Further, OT in relation to feeding behavior acts not only by maintaining homeostasis but also through the feelings of reward and hedonism. Olszewski et al [45] concluded that OT, as a feeding inhibitor, maintains homeostasis and is capable of mitigating some aspects of feeding behavior and energy metabolism. In addition, it was reported that feeding behavior induced serum OT release in dogs and sows [46], and dairy cows [47,48]. Though the mechanism of feeding-induced OT release is still unknown, it is a popular hypothesis that feeding behavior is controlled by not only the homeostatic system but also by the reward and hedonic system [49]. Researchers speculated two possibilities: one is that vagal fibers (secreting cholecystokinin, somatostatin, glucagon, and thyrotropin-releasing hormone) activate the brain [50], and the other is that sensory nerves in the oral mucosa are activated during feeding [42]. Sinomiya et al [51] reported that serum OT concentration tended to be higher in grazing cows than housing cows. It might be reasonable to suppose that the grazing system leads to more feeding and chewing behavior and more comfort to cows than the housing system, and that this increased comfort induces serum OT release in cows. Although it is difficult to conclude that the grazing system is more beneficial to animals than the housing system [52], animal welfare might be improved under the grazing system.

On the other hand, serum OT concentrations differed among individual calves, and the individual difference was stable in the same rearing system for several months [53]. However, different rearing systems may cause divergent physiological changes. In rats, chronic stress of repeated unavoidable stress induced an escape and a decrease in extra neuronal dopamine basal concentration [54]. Chronic stress of long term tethered housing pigs induced a greater increase in the adreno-corticotropic-hormone and cortisol than in loose-housed pigs [55]. Hence, physiological characteristics might be changed by the use of a long-term rearing system. OT also has a long-term effect. It was reported that intracerebroventricular OT administration decreased blood pressure and was effective for 8 days after the last injection [56], increased nociceptive thresholds for 3 weeks after the last injection of 10 days after a previous 5-day treatment [57], and decreased corticosterone for 10 days after the last injection of a regime administered once a day for 5 days [58] in rats. As mentioned above, manual brush- 
ing [59] suckling [42,33], and massaging [31] caused instantaneous increases in serum OT concentration. Thus, we consider that, if animals are reared in a long-term comfortable state, are provided stimuli such as brushing and massaging, and are reared under natural suckling or grazing rearing systems, they would have a higher basal OT concentration, which in turn might induce more positive normal behavior.

However, studies about serum OT have a limitation. As is well known, OT is produced in the supraoptic and paraventricular nuclei of the hypothalamus. However, it is difficult to directly inject OT into the brain and measure OT concentration in the brain in both humans and larger domestic animals. Administering OT via intranasal spray into the brain and measuring OT concentration in serum might solve these problems, and has been widely employed in many studies in humans and some domestic animals, although these studies have been criticized by Churchland and Winkielman [60]. They claim that the blood-brain barrier might prevent OT pullout from the blood into the brain, and that OT in the brain has a short life, and intranasal OT might hence not reach the OT receptor sites in the brain. In particular, it is of increasing importance to clarify the relationship of brain and serum OT concentrations. A few studies exist on serum OT, such as the study examining the feedback effects of OT mentioned above, facilitating OT release in both brain and blood $[39,20]$, as well as the studies demonstration that feeding behavior induces increases in serum OT [46-48]. Although studies on nasal administration of OT continue to be criticized, it may be considered certain that the relationship of OT with positive social behavior is mutually beneficial to some extent-the higher the OT concentration (modulated by general management practices, such as natural suckling, maternal care, and grazing; enrichment, such as manual brushing; and intranasal administration) the greater the degree of induction of positive social behavior, which in turn increases $\mathrm{OT}$ concentration.

\section{CONCLUSION}

OT is a key indicator of good welfare, enhancing pleasure, suppressing stress, and stimulating its own release. The release of OT is related to the performance of positive normal behavior, and enrichment, such as brushing, which can further accelerate OT secretion. Hence, studies on the welfare of farm animal should pay more attention to methods to improve management practices to induce OT and hence improve the comfort and stress tolerance of the animals, and not merely focus on how to control stress in farm animals.

\section{CONFLICT OF INTEREST}

We certify that there is no conflict of interest with any financial organization regarding the material discussed in the manuscript.

\section{ACKNOWLEDGMENTS}

This review was supported by the Laboratory of Land Ecology, Graduate School of Agricultural Science, Tohoku University. We would especially like to thank Assistant Professor Yu Yoshihara and Professor Shin-ichiro Ogura from the Laboratory of Land Ecology, and Associate Professor Sang-gun Roh from the Laboratory of Animal Physiology, Tohoku University.

\section{REFERENCES}

1. Mason G, Rushen J. 2008. Stereotypic Animal Behavior: Fundamentals and Applications to Welfare. 2nd edn. Trowbridge, UK: Cromwell press; 2007.

2. Lay DCJ, Friend TH, Grissom KK, Bowers CL, Mal ME. Effects of freeze or hot-iron branding of angus calves on some physiological and behavioral indicators of stress. Appl Anim Behav Sci 1992;33:137-47.

3. Duncan IJH, Olsson IAS. 2001. Environmental enrichment: from flawed concept to pseudo-science. In: Proceedings of 35th International Congress of the ISAE 2009. 2009 September 25-27, Vienna Austria: University of Natural Resources and Applied Life Sciences, pp. 73.

4. Viveros OH, Arqueros L, Kirshner N. Release of catecholamines and dopamine- $\beta$-oxidase from the adrenal medulla. Life Sci 1968; 7:609-18.

5. Bernard BB, Shore PA. A Concept for a role of serotonin and norepinepinphrine as chemical mediators in the brain. Ann NY Acad Sci 1957;66:631-42.

6. Bloom F, Segal D, Ling N, Guillemin R. Endorphins: profound behavioral effects in rats suggest new etiological factors in mental illness. Science 1976;194:630-2.

7. Uvnäs-Moberg K. Oxytocin may mediate the benefits of positive social interaction and emotions. Psychoneuroendocrinology 1998; 23:819-35.

8. Ross HE, Young LJ. Oxytocin and neural mechanisms regulating social cognition and affiliative behavior. Front Neuroendocrinol 2009;30:534-47.

9. Boissy A, Manteuffel G, Jensen MB, Moe RO, Spruijt B, Keeling LJ, Winckler C, Forkman B, Dimitrov I, Langbein J. Assessment of positive emotions in animals to improve their welfare. Physiol Behav 2007;92:375-97.

10. Broom DM, Fraser AF. Direct measures of good welfare In: Domestic Animal Behaviour and Welfare: Welfare Assessment. 4th edn. London, UK: Cambridge University Press; 2007.

11. Fraser D, Weary DM, Pajor EA, Milligan BN. A scientific conception of animal welfare that reflects ethical concerns. Anim Welf 1997; 6:187-205.

12. Carenzi C, Verga M. Animal welfare: Review of the scientific concept and definition. Ital J Anim Sci 2007;8:21-30.

13. Rushen J, de Passillé AMB. The scientific assessment of the impact of housing on animal welfare: A critical review. Can J Anim Sci 
1992;72:721-43.

14. Webster AJF. 2001. Farm animal welfare: the five freedoms and the free market. Vet J 2001;161:229-37.

15. Schwartzkopf-Genswein KS, Faucitano L, Dadgar S, et al. Road transport of cattle, swine and poultry in North America and its impact on animal welfare, carcass and meat quality: A review. Meat Sci 2012;92:227-43.

16. Argiolas A, Gessa GL. Central functions of oxytocin. Neurosci Biobehav Rev 1991;15:217-31.

17. Pedersen CA, Prange Jr AJ. Induction of maternal behavior in virgin rats after intracerebroventricular administration of oxytocin. Proc Natl Acad Sci USA 1979;76:6661-5.

18. Stamatadis A, Kalpachidou T, Raftogianni A, Zografou E, Tzanou A, Pondiki S, Stylianopoulou F. Rat dams exposed repeatedly to a daily brief separation from the pups exhibit increased maternal behavior, decreased anxiety and altered levels of receptors for estrogens (ERa, ER $\beta$ ), oxytocin and serotonin (5-HT1A) in their brain. Psychoneuroendocrinology 2015;52:212-28.

19. Pedersen CA, Caldwell JD, Peterson G, Walker CH, Mason GA. Oxytocin activation of maternal behavior in the rat. Anim. NY Acad Sci 1992;652:58-69.

20. Da CAP, Guevara-Guzman RG, Ohkura S, Goode JA, Kendrick $\mathrm{KM}$. The role of oxytocin release in the paraventricular nucleus in the control of maternal behavior in the sheep. J Neuroendocrinol 1996;8:163-77.

21. Williams GL, Gazal OS, Leshin LS, Stanko RL, Anderson LL. Physiological regulation of maternal behavior in heifers: roles of genital stimulation, intracerebral oxytocin release, and ovarian steroids. Biol Reprod 2001;65:295-300.

22. Winslow JT, Hastings N, Carter CS, Harbaugh CR, Insel TR. A role for central vasopressin in pair bonding in monogamous prairie voles. Nature 1993;365:545-8.

23. Babygirija R, Cerjak D, Yoshimoto S, et al. Affiliative behavior attenuates stress responses of GI tract via up-regulating hypothalamic oxytocin expression. Auton Neurosci 2012;169:28-33.

24. Dhakar MB, Rich ME, Reno EL, Lee H, Caldwell HK. Heightened aggressive behavior in mice with lifelong versus postweaning knockout of the oxytocin receptor. Horm Behav 2012;62:86-92.

25. Amico JA, Vollmer RR, Karam JR, et al. Centrally administered oxytocin elicits exaggerated grooming in oxytocin null mice. Pharmacol Biochem Behav 2004;78:333-9.

26. Winslow JT, Insel TR. Social status in pairs of male squirrel monkeys determines the behavioral response to central oxytocin administration. J Neurosci 1991;11:2032-8.

27. Svanidze MD, Bukiya NG, Butskhrikidze MP. Effect of oxytocin on the emotional state and behavior of rats under stress conditions. Neurophysiology 2012;43:365-8.

28. Dunbar RIM. The social role of touch in humans and primates: Behavioural function and neurobiological mechanisms. Neurosci Biobehav Rev 2010;34:260-8.

29. Sato S, Tarumizu K, Hatae K. The influence of social factors on allogrooming in cows. Appl Anim Behav Sci 1993;38:235-44.
30. Kurosawa M, Lundeberg T, Agren G, Lund I, Uvnäs-Moberg K. Massage-like stroking of the abdomen lowers blood pressure in anesthetized rats: influence of oxytocin. J Auton Nerv Syst 1995; 56:26-30.

31. Morhenn VB, Park JW, Piper E, Zak PJ. Monetary sacrifice among strangers is mediated by endogenous oxytocin release after physical contact. Evol Hum Behav 2008;29:375-83.

32. Holt-Lunstad J, Birmingham WA, Light KC. Influence of a "Warm Touch" support enhancement intervention among married couples on ambulatory blood pressure, oxytocin, alpha amylase, and cortisol. Psychosom Med 2008;70:976-85.

33. Chen S, Tanaka S, Ogura S, Roh S, Sato S. Effect of suckling systems on serum oxytocin and cortisol concentrations and behavior to a novel object in beef calves. Asian Australas J Anim Sci 2015;28: 1662-8.

34. Algers B, Rojanasthien B, Uvnäs-Moberg K. The relationship between teat stimulation, oxytocin release and grunting rate in the sow during nursing. Appl Anim Behav Sci 1990;26:267-76.

35. Parker KJ, Buckmaster CL, Schatzberg AF, Lyons DM. Intranasal oxytocin administration attenuates the ACTH stress response in monkeys. Psychoneuroendocrinology 2005;30:924-9.

36. Yayou K, Ito S, Kasuya E, et al. Intracerebroventricularly administered oxytocin attenuated cortisol secretion, but not behavioral responses, during isolation in Holstein steers. J Vet Med Sci 2008; 70:665-71.

37. Uvnäs-Moberg K, Ahienius S, Hillegaart V, Alster P. High doses of oxytocin cause sedation and low doses cause an anxiolytic-like effect in male rats. Pharmacol Biochem Behav 1994;49:101-6.

38. Windle RJ, Shanks N, Lightman SL, Ingram CD. Central oxytocin administration reduces stress-induced corticosterone release and anxiety behavior in rats. Endocrinology 1997;138:2829-34.

39. Moos F, Freund-Mercier MJ, Guerné Y, et al. Release of oxytocin and vasopressin by magnocellular nuclei in vitro: Specific facilitatory effect of oxytocin on its own release. J Endocrinol 1984;102:6372.

40. Neumann I, Koehler E, Landgraf R, Summy-Long J. An oxytocin receptor antagonist infused into the supraoptic nucleus attenuates intranuclear and peripheral release of oxytocin during suckling in conscious rats. Endocrinology 1994;134:141-8.

41. Mellor DJ. Animal emotions, behaviour and the promotion of positive welfare states. New Zealand Vet J 2011;60: 1-8.

42. Lupoli B, Johansson B, Uvnäs-Moberg K, Svennersten-Sjaunja K. Effect of suckling on the release of oxytocin, prolactin, cortisol, gastrin, cholecystokinin, somatostatin and insulin in dairy cows and their calves. J Dairy Res 2001;68:175-87.

43. Odendaal JS, Meintjes RA. Neurophysiological correlates of affiliative behaviour between humans and dogs. Vet J 2003;165: 296-301.

44. Krohn CC. Effects of different suckling systems on milk production, udder health, reproduction, calf growth and some behavioural aspects in high producing dairy cows - A review. Appl Anim Behav Sci 2001;72:271-80. 
45. Olszewski PK, Klockars A, Schiöth HB, Levine AS. Oxytocin as feeding inhibitor: Maintaining homeostasis in consummatory behavior. Phamacol Biochem Behav 2010;97:47-54.

46. Uvnäs-Moberg K, Stock S, Eriksson M, et al. Plasma levels of oxytocin increase in response to suckling and feeding in dogs and sows. Acta Physiol Scand 1985;124:391-8.

47. Svennersten K, Nelson L, Uvnäs-Moberg K. Feeding-induced oxytocin release in dairy cows. Acta Physiol Scand 1990;140:2956.

48. Lindström T, Redbo I, Uvnäs-Moberg K. Plasma oxytocin and cortisol concentrations in dairy cows in relation to feeding duration and rumen fill. Physiol Behav 2001;72:73-81.

49. Ginane D, Bonnet M, Baumont R, Revell DK. Feeding behaviour in ruminants: A consequence of interactions a reward system and the regulation of metabolic homeostasis. Anim Prod Sci 2015;55: 247-60.

50. Arletti R, Benelli A, Bertolini A. Influence of oxytocin on feeding behavior in the rat. Peptides 1989;10:89-93.

51. Shinomiya T, Sato K, Tanaka S, Ninomiya S, and Sato S. Comparing welfare of dairy cows in the grazing system and the housing system. In: Proceedings of 14th AAAP Animal Science Congress 2010. 2010 Augest 23-27, Pingtung, Taiwan. 3:2182-2185.

52. Charlton GL, Rutter SM, East M, Sinclair LA. Preference of dairy cows: Indoor cubicle housing with access to a total mixed ration vs. access to pasture. Appl. Anim Behav Sci 2011;130:1-9.

53. Chen S, Tanka S, Oyakawa C, Roh S, Sato S. Individual difference in serum oxytocin concentrations of calves and the correlation with those in dams. Anim Sci J 2014;85:53-57.

54. Mangiavacchi S, Masi F, Scheggi S, et al. Long-term behavioral and neurochemical effects of chronic stress exposure in rat. J Neurochem 2001;79:1113-21.

55. Janssens CJ, Helmond FA, Loyens LW, Schouten WG, Wiegant VM. Chronic stress increases the opioid-mediated inhibition of the pituitary-adrenocortical response to acute stress in pigs. Endocrinology 1995;136:1468-73.

56. Petersson M, Alster P, Lundeberg T, Uvnäs-Moberg K. Oxytocin causes a long-term decrease of blood pressure in female and male rats. Physiol Behav 1996;60:1311-5.

57. Petersson M, Alster P, Lundeberg T, Uvnäs-Moberg K. Oxytocin increases nociceptive thresholds in a long-term perspective in female and male rats. Neurosci Lett 1996;212:87-90.

58. Petersson M, Hulting AL, Uvnäs-Moberg K. Oxytocin causes a sustained decrease in plasma levels of corticosterone in rats. Neurosci Lett 1999;264:41-4.

59. Chen S, Tanaka S, Roh S, Sato S. Factors affecting oxytocin concentration and relationship between oxytocin concentration and affiliative behaviour. In: Proceedings of the 48th International Congress of the International Society for Applied Ethology 2014; 2014 July 27-Augest 2, Vitoria-Gasteiz, Spain. pp.137.

60. Churchland PS, Winkielman P. Modulating social behavior with oxytocin: How does it work? What does it mean? Horm Behav 2012;61:392-9. 